\title{
PRINCIPIOS DE LA CONSTITUCIÓN AMBIENTAL
}

\author{
PRINCIPLES OF THE ENVIRONMENTAL CONSTITUTION
}

César Landa

Profesor de Derecho Constitucional de la Pontificia Universidad Católica del Perú y en la Universidad Nacional Mayor de San Marcos. Ex Presidente del Tribunal Constitucional.

E-mail: clanda@pucp.edu.pe

Convidado

Doi: $10.5585 /$ rdb.v16i7.533

RESUMEN: La relación entre la sociedad y el Estado ha cambiado de tal manera que una vez que la libertad era sinónimo de derecho fundamental, ahora hay que acoplarse con ciertos valores sociales, así como, más recientemente, los valores ambientales. El crecimiento económico unido a otras circunstancias tales como el calentamiento global y la degradación del medio ambiente, ha incorporado la preocupación del Estado, a través de la actuación de las organizaciones internacionales especializadas. Lo que se conoce como Constitución ambiental contemporánea, objeto de este artículo, se manifiesta en forma de principios internacionales y de derechos fundamentales (o derechos humanos del medio ambiente) y no necesariamente a través de leyes, todo para hacer a escapar de las formalidades legalistas. Aquí se analizará el contenido de dichos principios (desarrollo sostenible, precaución y prevención) a través de la jurisprudencia de la Corte Constitucional y el Tribunal de Vigilancia del Medio Ambiente, de Perú, así como las jurisprudencias incidentales de la Corte Interamericana de derechos Humanos.

Palavras-chave: Principios Ambientales. Principio de Desarrollo Sostenible. Generaciones Futuras. Principio de Precaución. Principio de Prevención. Constitución Ambiental.

ABSTRACT: The relationship between society and the State has been changing in such a way that once freedom was synonymous of fundamental rights and now that there is to be coupled with certain social values, as well as, more recently, environmental values. Economic growth coupled with other circumstances such as global warming and environmental degradation has incorporated State concerns, through the work of specialized international organizations. What became known as Contemporary Environmental Constitution, subject of this article, is manifested in the form of international principles and fundamental rights (or environment human rights) and not necessarily through laws, in order to escape from legalistic formalities. This article shall analyze the content of such principles (sustainable development, precaution and prevention) through jurisprudence of the Constitutional Court and the Environmental Surveillance Court, both courts in Peru, as well as the incidental jurisprudence of the InterAmerican Court of Human rights.

Keywords: Environmental Principles. Principle of Sustainable Development. Future Generations. Principle of Precaution. Principle of Prevention. Environmental Constitution. 
SUMÁRIO: Presentación. 1. Principios Ambientales. 1.1. Principio de Desarrollo Sostenible y Generaciones Futuras. 1.2. Principio de Precaución. 1.3. Principio de Prevención. Conclusión. Referencias.

SUMMARY: Introduction. 1. Environmental Principles. 1.1. Principle of Sustainable Development and Future Generations. 1.2. Principle of Precaution. 1.3. Principle of Prevention. Conclusion. Literature.

\section{PRESENTACIÓN}

En sus orígenes el Estado de Derecho construido por el pensamiento liberal se fundó en base a la relación entre la sociedad y el Estado; es decir que la forma de organización del poder mediante su división era una garantía para las libertades humanas. Esa relación entre la autoridad y los ciudadanos era básicamente jurídica y política; de modo que la ley vertebraba al Estado de Derecho y la libertad quedaba garantizada a través de la igualdad de todos ante ley.

Pero, con el surgimiento de la "cuestión social", expresada en las revueltas y revoluciones contra el poder, por las miserias que generaba el capitalismo salvaje, el Estado de Derecho basado en la ley se transformó hacia un Estado de Derecho basado en la Constitución, incorporando a la relación entre la sociedad y el Estado, a la economía. Por eso, el nuevo Estado de bienestar asumió las cargas sociales no resueltas por el libre mercado y también distribuyó responsabilidades a los grandes propietarios, a través de derechos sociales y económicos para los trabajadores y demás desvalidos.

Sin embargo, el crecimiento económico asumido como base para superar la pobreza se basó en la destrucción de la naturaleza. Por eso, ante el calentamiento global debido a la degradación de las condiciones ambientales, que empezaron a poner en peligro no solo a las especies - animales y vegetales -, a los recursos naturales - renovables y no renovables - , y, también a la calidad de vida humana ${ }^{1}$, se incorpora a la tríada de sociedad, Estado y economía, al medio ambiente.

Así, el postulado contemporáneo de la "Constitución Ambiental o Ecológica", se manifiesta en forma de principios y derechos fundamentales; pero, que no pueden ser implementados plenamente desde una perspectiva legalista, por la estreches de su sujeción al formalismo de la ley, sino que requiere de una concepción más integradora desde la Constitución y los tratados internacionales; a través de la jurisprudencia nacional e internacional.

Bajo tales supuestos, siguiendo las tesis de Saladin y Häberle, es necesario analizar si se viene construyendo un modelo de justicia ambiental, basada en tres principios: primero, la justicia intrageneracional - intragenerationelle Gerechtigkeit, de quienes comparten un mismo período de vida; segundo, la justicia como responsabilidad con las generaciones futuras Intergenerationelle Gerechtigkeit; y, tercero, la justicia como el respeto humano a su medio ambiente natural - natürlichen Mitwelt ${ }^{2}$.

\footnotetext{
${ }^{1}$ Cfr. Acuerdo de Paris sobre Cambio Climático de la Convención Marco de las Naciones Unidas sobre el Cambio Climático. En http://unfccc.int/resource/docs/2015/cop21/spa/109s.pdf. Visitado el 1 de octubre de 2016; asimismo, PROGRAMA DE NACIONES UnidAS PARA El MEDIO AMBIENTE. Medio ambiente para el futuro que queremos. En http://www.pnuma.org/geo/geo5/GEO\%205\%20ESPANOL \%202013\%20WEB.pdf. Visitado el 3 de octubre de 2016, y; AL GoRE, Una verdad incómoda. En http://es.slideshare.net/edau1/una-verdad-incmoda-al-gore. Visitado el 3 de octubre de 2016.

${ }^{2}$ Saladin, Peter. Wozu noch Saaten? Berna: Verlag Stämpfli+Cie AG, 1995, pp. 93 ss.; HÄBerle, Peter. "Ein Verfassungsrecht für künftige Generationen. Die "andere" Form des Gesellschaftsvertrages: der Generationenvertrag". En Verfassung, Theorie und Praxis des Sozialstaats. Festschrift für Hans F. Zacher zum 70. Geburtstag, 1998, pp. 215-233.
} 
Entonces, es en el marco de los principios de la llamada "Constitución Ambiental" que se va analizar el desarrollo de los principios ambientales, a través de la jurisprudencia del Tribunal Constitucional, del Tribunal de Fiscalización Ambiental y la jurisprudencia incidental de la Corte Interamericana de Derechos Humanos. Ello nos permitirá entender cuan presente se encuentra el tratamiento y la protección del derecho de la naturaleza en nuestro país; por cuanto, constituye un problema vital para el Estado mantener la dignidad humana de hoy y de las generaciones futuras, a través de obligaciones ambientales objetivas para el propio Estado y para los particulares, a fin de asegurar la protección de los derechos fundamentales a un medio ambiente adecuado para todos las personas.

\section{PRINCIPIOS AMBIENTALES}

Los principios son disposiciones normativas generales de todo cuerpo jurídico que cumplen tres funciones: primero, como "metanormas", esto es normas abiertas con las cuales se puede concretizar a las normas específicas de un código; segundo, como normas "hermenéuticas", porque ante el vacío o deficiencia de un código permiten suprimir las lagunas normativas, y; tercero, como normas "teleológicas" orientadas a la concretización y optimización de los principios materiales.

En ese entendido, para el Tribunal Constitucional:

[...] la tutela del medio ambiente se encuentra regulada en nuestra "Constitución Ecológica”, que no es otra cosa que el conjunto de disposiciones [o principios] de nuestra Constitución que fijan las relaciones entre el individuo, la sociedad y el medio ambiente, tema que ocupa un lugar medular en nuestra Ley Fundamental. (Añadido nuestro) ${ }^{3}$.

Así, la protección al medio ambiente, por un lado, es un principio que vincula a todo el ordenamiento jurídico, dado que es una obligación del Estado proteger las recursos naturales que son de la Nación; por otro lado, es un derecho fundamental de todas las personas a gozar de un ambiente adecuado, que se hace exigible judicialmente, y: finalmente, es un mandato a implementar la "Constitución Ambiental" de la cual se originan obligaciones para las autoridades y los particulares ${ }^{4}$.

De modo que, la Constitución Ambiental cuenta con un conjunto de principios previstos en la Norma Suprema y tratados internacionales, que se recogen en la Ley General del Ambiente, Ley $\mathrm{N}^{\circ} 28611(2015)^{5}$, que ordenan las relaciones entre la persona, la sociedad y el medio ambiente, a través de principios, de los cuales se analiza tres que han sido recogidos jurisprudencialmente, como los siguientes:

\subsection{Principio de Desarrollo sostenible y Generaciones Futuras}

En la medida que la protección de la persona humana y el respeto de su dignidad es el fin supremo de la sociedad, la economía y del Estado, el desarrollo sostenible busca mejorar la calidad de vida de los seres humanos, sin perjudicar los ciclos de la naturaleza. Es decir se trata de mantener la dinámica de la biodiversidad para satisfacer las necesidades de la población

\footnotetext{
${ }^{3}$ Tribunal Constitucional. Sentencia recaída en el Expediente N 3610-2008-AA. FJ. 33.

${ }^{4}$ Tribunal Constitucional. Sentencia recaída en el Expediente 03343-2007-PA. FJ. 6, que se remite al fallo de la Corte Constitucional de Colombia. Sentencia T-760/07.

${ }^{5}$ La Ley $N^{\circ} 28611$, consagra en su Título Preliminar, los principios de sostenibilidad, prevención, precautorio, de internacionalización de costos, de responsabilidad ambiental, de equidad y de gobernanza ambiental; además del derecho a la justicia ambiental, participación y acceso a la información.
} 
presente y futura. El progreso social sin daño ambiental es posible en el marco de la Constitución Ambiental, para lo cual se debe articular el modelo de explotación económica con el derecho a un ambiente equilibrado y adecuado ${ }^{6}$.

Como la Constitución prescribe que el Estado determina la política nacional del ambiente, así como promueve el uso sostenible de sus recursos naturales y especialmente el desarrollo sostenible de la Amazonía (artículos 67 y 69); el Estado está obligado a promover la conservación de la diversidad biológica y de las áreas naturales protegidas (artículo 68). De esta forma, nuestros recursos naturales pueden ser aprovechados de forma racional y razonable, evitando su extinción o disminución a largo plazo; preservando la satisfacción de las necesidades de las venideras y futuras generaciones.

Sobre el particular, a partir del Informe Bruntland de la Comisión Mundial Para el Medio Ambiente y el Desarrollo de la ONU (1987) se busca superar la confrontación entre el desarrollo económico y la protección del medio ambiente; mediante la incorporación del modelo de desarrollo sostenible (o sustentable); definido como "aquel que satisface las necesidades del presente sin comprometer las necesidades de las futuras generaciones". Para lo cual los Estados deben orientar las inversiones y los cambios tecnológicos, hacia un modelo que integre a la naturaleza, debido a que el "desarrollo sostenible no es un estado concreto, sino un proceso de cambio en donde la explotación de recursos, la dirección de las inversiones, la orientación de los desarrollos tecnológicos y los cambios institucionales, deben ser consistentes con el futuro así como con el presente"7.

En ese sentido, a juicio del Tribunal Constitucional el legado ambiental no se agota con asegurar el bienestar general de la población del presente, sino que:

En lo que respecta al principio de desarrollo sostenible o sustentable, constituye una pauta basilar para que la gestión humana sea capaz de generar una mayor calidad y condiciones de vida en beneficio de la población actual, pero manteniendo la potencialidad del ambiente para satisfacer las necesidades y las aspiraciones de vida de las generaciones futuras. Por ende, propugna que la utilización de los bienes ambientales para el consumo no se "financien" incurriendo en "deudas" sociales para el porvenir".

Asimismo, cabe recordar que la Constitución: por un lado, reconoce que toda persona tiene derecho a gozar de un ambiente equilibrado y adecuado al desarrollo de su vida, así como, a la paz, a la tranquilidad, al disfrute del tiempo libre y al descanso (artículo 2-22), y: por otro lado, establece que los recursos naturales, renovables y no renovables, son patrimonio de la Nación; pero, el Estado es soberano en su aprovechamiento, por eso los otorga en concesión de conformidad con una ley orgánica (artículo 66). De modo que, la explotación de los recursos naturales, particularmente los no renovables-, deben ser objeto de un aprovechamiento razonable y sostenible, en favor de la colectividad en general, y, de la población local en particular; correspondiendo así a las circunscripciones recibir una participación adecuada del Estado por las rentas e ingresos obtenidos por la explotación de los recursos naturales en cada zona en calidad de canon (artículo 77 in fine).

Ello supone plantear la responsabilidad social de las empresas con el desarrollo sostenible; así como, la inversión y el desarrollo, en el marco de la Constitución ${ }^{9}$; por cuanto, no se trata de contraponer: de un lado, el progreso y las posibilidades de desarrollo a partir de

\footnotetext{
${ }^{6}$ Foy VALENCIA, Pierre. "Constitucionalidad del Desarrollo Sostenible". Lima, marzo 2014, inédito.

${ }^{7}$ INFORME BRUNTLAND. https://desarrollosostenible.wordpress.com/2006/09/27/informe-brundtl and/. Visitado el 10 de octubre de 2016.

${ }^{8}$ TRIBUNAL CONSTITUCIONAL. Sentencia recaída en el Expediente No 0048-2004-PI/TC. FJ 19.

9 Tribunal Constitucional. Sentencia recaída en el Expediente $\mathrm{N}^{\circ}$ 06316-2008-PA/TC. Voto singular del Magistrado Landa Arroyo, FJ 22-38.
}

Revista de Direito Brasileira | São Paulo, SP | v. 16 | n. 7 | p. 412 - 427 | Jan./Abr. 2017 
fomentar la iniciativa privada para la explotación de los recursos naturales, y, de otro lado, un conservacionismo inmovilista del medio ambiente. En efecto, la sostenibilidad supone la responsabilidad social de las empresas con el medio ambiente y la racional explotación de los recursos naturales; si caer en u fundamentalismo ecológico ${ }^{10}$.

En ese entendido, el Tribunal Constitucional ha señalado que:

Como se aprecia, la perspectiva del desarrollo sostenible busca equilibrar el esquema de la economía social de mercado con el derecho a vivir en un ambiente equilibrado y adecuado. Es una maximización de las ganancias o utilidad frente a la calidad del entorno que sufre el desgaste de la actividad económica. En tal sentido, con el principio sostenibilidad (artículo V de la Ley General del Ambiente) se pretende modular esta actividad económica a la preservación del ambiente, el mismo que tendrá que servir de soporte vital también para las generaciones venideras. Así, los derechos de las actuales generaciones no deben ser la ruina de las aspiraciones de las generaciones futuras ${ }^{11}$.

Por su parte, la Corte Interamericana de Derechos Humanos (Corte IDH) ha destacado incidentalmente en sus sentencias referidas a la protección de los territorios de los pueblos indígenas, en base al derecho de propiedad; que:

[....] para que la exploración o extracción de recursos naturales en los territorios ancestrales no impliquen una denegación de la subsistencia del pueblo indígena como tal, el Estado debe cumplir con las siguientes salvaguardias: i) efectuar un proceso adecuado y participativo que garantice su derecho a la consulta, en particular, entre otros supuestos, en casos de planes de desarrollo o de inversión a gran escala; ii) la realización de un estudio de impacto ambiental; y iii) en su caso, compartir razonablemente los beneficios que se produzcan de la explotación de los recursos naturales (como una forma de justa indemnización exigida por el artículo 21 de la Convención), según lo que la propia comunidad determine y resuelva respecto de quiénes serían los beneficiarios de tal compensación según sus costumbres y tradiciones. ${ }^{12}$ (Subrayado nuestro).

En consecuencia, cabe señalar que no se trata de preservar exclusivamente el legado ambiental para las nuevas generaciones, sino en el marco de una sociedad multicultural como la nuestra. Lo cual permite proteger los bienes ambientales de los peligros de su existencia, a través de medidas de control que debe implementar el Estado.

Más aún, si, con el nuevo mileno, la sociedad del riesgo es una nueva forma de existencia de nuestra civilización, ya no como un fenómeno eventual ni esporádico, sino regular y permanente $^{13}$; entonces, parece razonable constitucionalizar los principios de precaución y de prevención. Ello, debido a que las inversiones económicas basan sus costos y beneficios considerando los riesgos existentes; pero, dentro de estos no ha existido la obligación de considerar la eventual afectación a la naturaleza y al medio ambiente.

\footnotetext{
${ }^{10}$ HÄBERLE, Peter. Retos actuales del Estado Constitucional. Oñati: IVAP, 1996, p. 144.

${ }^{11}$ Tribunal Constitucional. Sentencia recaída en el Expediente Nº .03343-2007-PA/TC. FJ 14.

${ }^{12}$ CoRTe IDH. Caso Pueblo Indígena Kiwcha de Sarayaku vs. Ecuador. Sentencia de 27 de junio de 2012, párrafo 157; asimismo, Caso del Pueblo Saramaka vs. Surinam. Sentencia del 28 de noviembre de 2007, párrafo 158.

${ }^{13}$ BECK, Ulrich. La sociedad del riesgo: hacia una nueva modernidad. Bracelona: Paidós, 2001.
} 
Por eso, el Estado con criterios de racionalidad está buscando combinar efectividad con la necesidad de prevenir graves riesgos para el medio ambiente ${ }^{14}$; para lo cual la Ley General del Ambiente ha establecido el principio precautorio y el principio de prevención, que por su tratamiento jurisprudencial constitucional a continuación se analiza.

\subsection{Principio de Precaución}

La definición inicial del principio precautorio o de precaución se adoptó en la Conferencia de las Naciones Unidas sobre el Medio Ambiente y el Desarrollo de Rio de Janeiro en 1992, al señalar que: "Cuando haya peligro de daño grave o irreversible, la falta de certeza científica absoluta no deberá utilizarse como razón para postergar la adopción de medidas eficaces en función de los costos para impedir la degradación del medio ambiente"15.

No obstante, el principio precautorio tiene un origen alemán (Vorsorgeprinzip). Se incluyó en una norma legal sobre saneamiento del aire en 1974; pero, hoy se ha extendido como un criterio de protección del derecho al medio ambiente sano y equilibrado ${ }^{16}$. Por eso, desde sus inicios el Ministerio Federal del Interior de la República Federal Alemana señaló que el principio precautorio involucraba la detección a tiempo de los riesgos para la salud y el medio ambiente, a través de investigaciones completas sobre la materia ${ }^{17}$.

De hecho, en el ámbito europeo, también se entiende que el riesgo de una actividad y la aplicación del principio precautorio tienen alcances no solo en el ámbito ambiental, sino además en el ámbito de lo sanitario; así, se ha indicado que: "el principio de precaución sólo puede aplicarse (...) cuando exista un riesgo, y en particular un riesgo para la salud humana, que sin estar basado en meras hipótesis no verificadas científicamente, aún no ha podido ser plenamente demostrado" 18 .

En el ordenamiento jurídico peruano la Ley General del Ambiente, Ley $\mathrm{N}^{\circ} 28611$, establece en el Título Preliminar que: "Cuando haya peligro de daño grave o irreversible, la falta de certeza absoluta no debe utilizarse como razón para postergar la adopción de medidas eficaces y eficientes para impedir la degradación del ambiente" (Artículo VII).

En ese entendido, el Tribunal Constitucional ha señalado que el instituto de la precaución es un principio que determina o activa un principio orientado a que la tutela de un medio ambiente adecuado y equilibrado frente a situaciones de amenaza de un daño al medio ambiente, en las que existe falta de certeza científica sobre sus causas y efectos no se pueda afectar. ${ }^{19}$ En concreto, se ha indicado lo siguiente:

Al principio precautorio se le pueden reconocer algunos elementos. Entre ellos: a) la existencia de una amenaza, un peligro o riesgo de un daño; b) la existencia

\footnotetext{
${ }^{14}$ EMBID TELlo, Antonio. Retos jurídicos de la radiación no ionizante, Tesis doctoral, Madrid, Universidad Carlos III, disponible en: http://e-archivo.uc3m.es/bitstream/handle/10016/7622 /antonio embid tesis.pdf;jsessionid=D4B659855A6F6E75F9B68B6ECEEB7FEC?sequence=1. Visitado el 10 de octubre de 2016.

${ }^{15}$ Conferencia de las Naciones Unidas sobre El Medio Ambiente Y el Desarrollo. Declaración de Rio sobre el Medio Ambiente y el Desarrollo. En http://www.pnuma.org/sociedad civil/reunion2013/docu mentos/STAKEHOLDER\%20PARTICIPATION/1992\%20Declaraci\%C3\%B3n\%20de\%20R\%C3\%ADo\%20Espa\% C3\%B1ol.pdf. Visitado el 10 de octubre de 2016.

16 En la actualidad también se aplica al ámbito del derecho a la salud y a la salud pública. Cfr. http://www.bioscience-bioethics.org/pdfs/NW.Public.Health.PP.2005.pdf. Visitado el 11 de octubre de 2016.

${ }^{17}$ COMisión Mundial de ÉTICA DEL CONOCIMIENTO CiEnTífiCO Y LA TECNOLOGía (COMEST). INFORME DEL GRUPO DE EXPERTOS SOBRE EL PRINCIPIO PRECAUTORIO. PARIS, UNESCO, 2005, p. 13. En http://unesdoc.unesco.org/ images/0013/001395/139578s.pdf. Visitado el 15 de octubre de 2016.

${ }^{18}$ Tribunal De Justicia DE LA COMUnidAD EuropeA - STPI. Caso Pfizer Animal Health, del 11 de septiembre de 2002, asunto T-13/99, o, caso Monsanto Agricultura Italia y otros, de 9 de septiembre de 2003, asunto C-236/00. En http://curia.europa.eu. Visitado 10 de octubre de 2016.

${ }^{19}$ TRIBUNAL CONSTITUCIONAL. Sentencia recaída en el expediente No 02005-2009-AA/TC. FJ 49.

Revista de Direito Brasileira | São Paulo, SP | v. 16 | n. 7 | p. 412 - 427 | Jan./Abr. 2017
} 
de una incertidumbre científica, por desconocimiento, por no haberse podido establecer evidencia convincente sobre la inocuidad del producto o actividad aun cuando las relaciones de causa-efecto entre éstas y un posible daño no sean absolutas, o incluso por una importante controversia en el mundo científico acerca de esos efectos en cuestión; y, c) la necesidad de adoptar acciones positivas para que el peligro o daño sea prevenido o para la protección del bien jurídico como la salud, el ambiente, la ecología, etc ${ }^{20}$.

Ahora bien, el principio precautorio se encuentra vinculado a la relación riesgobeneficio que se produce a raíz de las actividades económicas que se realizan sobre los recursos naturales y el medio ambiente; en razón de ello, se podría decir que en la medida que el riesgo se define como el potencial para la realización de consecuencias no deseadas, la precaución es el principio que se activa cuando se comprueba la existencia de aquel. En concreto, se puede señalar que este concepto prima facie no se aplica para gestionar el riesgo, sino para tratar de controlar las causas de su ocurrencia ${ }^{21}$.

Pero, la aplicación del principio se sustenta en determinados parámetros, que se pueden desprender de lo señalado por el Tribunal Constitucional y por el Grupo de Expertos de Naciones Unidas sobre el principio precautorio. En concreto, en el caso de la protección del medio ambiente, el principio precautorio debe aplicarse cuando: a) existe una incertidumbre acerca de la naturaleza y magnitud del daño; b) los motivos de incertidumbre sean plausibles o científicamente defendibles; c) el riesgo tenga consecuencias poco conocidas; d) los peligros sean inaceptables $^{22}$.

Asimismo, se puede afirmar que en la mejor doctrina se ha adoptado una postura minimalista en la que el principio precautorio solo se aplica en caso de riesgo inminente de graves daños irreversibles; a diferencia de la posición maximalista que busca alcanzar un nivel de riesgo "cero" a partir del cual la acción pública puede darse en condiciones incluso de ignorancia científica $^{23}$.

Ahora bien, conviene mencionar que en el caso peruano, inicialmente el Tribunal Constitucional aplicó la postura maximalista del principio de precaución. En el caso de las antenas Nextel, el primero de los fallos emitido en el año 2003, declaró fundada la demanda y el consiguiente retiro de las antenas de la Av. Prolongación Javier Prado, so pretexto del eventual peligro a la salud de la radiación de las antes retransmisoras de la telefonía móvil.

Respecto de la posible afectación del derecho a la salud y a un medio ambiente sano y adecuado a consecuencia de la propagación de ondas electromagnéticas, este Tribunal debe destacar que se trata de un tema en el que, desde un punto de vista científico, no existe actualmente consenso. Sí existe consenso, sin embargo, en que a través de la legislación correspondiente se establezca una serie de precauciones destinadas a evitar que la carencia de resultados satisfactorios en la investigación sobre el tema, no termine generando problemas irreversibles en la salud y el medio ambiente $[\ldots]^{24}$.

\footnotetext{
${ }^{20}$ Ibídem, FJ. 50.; asimismo, Tribunal Constitucional. Sentencia recaída en expediente $\mathrm{N}^{\circ}$ 4223-2006-PA/TC. FJ28.

${ }^{21}$ BEN LAZAAR, Soufiane. El principio de precaución en el derecho internacional del medio ambiente. Trabajo de Fin de Máster, Universidad Internacional de Andalucía, 2012, disponible en: http://dspace.unia.es/bitstream/handle/10334/2117/0256_BenLazaar.pdf?sequence=1. Revisado el 01 de octubre de 2016.

${ }^{22}$ COMEST..., op. cit., p. 14.

${ }^{23}$ Huerta GUERRERO, Luis. "Constitucionalización del derecho ambiental”. En Derecho PUCP 71. Lima: Fondo Editorial PUCP, 2013, pp. 498-501.

${ }^{24}$ TRIBUNAL CONSTITUCIONAL. Sentencia recaída en el Expediente No 0964-2002-AA/TC, FJ 11.
} Revista de Direito Brasileira | São Paulo, SP | v. 16 | n. 7 | p. 412 - 427 | Jan./Abr. 2017 
Sin embargo, esta postura cambió, ya que en el segundo de los fallos del caso Nextel emitido en el año 2007, la demanda que solicitaba el retiro de las Antenas Nextel se declaró infundada, ya que no existía acreditación de un riesgo razonable ni suficiente para la salud y el medio ambiente. En ese entendido, en este caso se adoptó una posición minimalista dado que no se había acreditado un riesgo real. Por eso, el Tribunal Constitucional sobre el principio precautorio estableció que:

En los fundamentos precedentes, se ha señalado que el principio precautorio se aplica ante la amenaza de un daño a la salud o medio ambiente y ante la falta de certeza científica sobre sus causas y efectos. Si bien el presupuesto esencial para la aplicación del principio precautorio es precisamente la falta de certeza científica -aun cuando no sea imprescindible demostrar plenamente la gravedad y realidad del riesgo-, sí resulta exigible que haya indicios razonables y suficientes de su existencia y que su entidad justifique la necesidad de adoptar medidas urgentes, proporcionales y razonables ${ }^{25}$.

De acuerdo con lo mencionado, la aplicación del principio precautorio debe ser proporcional, en ese sentido debe señalarse que una prohibición total de una actividad, no necesariamente es la respuesta más adecuada en todos los casos. En efecto, tal como lo ha señalado el Tribunal Constitucional: "no siempre la prohibición absoluta de determinada actividad es la única vía para alcanzar determinado grado de protección, pues, dependiendo del caso, el mismo puede ser alcanzado, mediante la reducción de la exposición al riesgo, con el establecimiento de mayores controles y la imposición de ciertas limitaciones"26.

Eventualmente en materia de otorgamiento de licencias y autorizaciones de ciertas actividades extractivas, este podría ser autorizado bajo ciertas condiciones o restricciones por el probable impacto de la actividad económica en la zona. En todo caso, en este ámbito también cobra especial relevancia la fiscalización y vigilancia, ya que por medio de ella se tendrá mayor información sobre los riesgos y daños de una actividad sobre el que no se tiene certeza científica.

Así, en el caso de la empresa Praxair Peru S.A., la Municipalidad de Bellavista (Callao) fue demanda por algunos pobladores que vivían colindantes a la empresa, debido a que en proceso del llenado de balones de oxígeno su chimenea exhalaba humos que perjudicaban la salud y la tranquilidad de los vecinos, pero, también demandaron al Municipio porque no cumplía con sus deberes de supervisar a dicho establecimientos que afectaba los derechos señalados. No obstante, el Tribunal Constitucional consideró que pese a no haber acreditado la relación de causalidad de lo denunciado:

[...] no exime a la entidad accionada del deber de fiscalizar las actividades que pudieran afectar el medio ambiente. Como ya ha sido señalado en el proceso de amparo seguido contra la empresa Praxair Perú S.A. (STC 3510-2003-AA, f. 19), "Este Tribunal no es ajeno al hecho de que si bien no existen elementos suficientes para que pueda emitirse una sentencia estimativa, dadas las actividades industriales de la accionada y su ubicación colindante con el Hospital Naval y la zona residencial de densidad media alta, se hace imprescindible, en atención a los principios de prevención y precaución, que el Estado adopte acciones positivas para asegurar la salud e integridad de la población asentada alrededor de la planta industrial de la demandada, y que esta dé estricto cumplimiento a la normativa ambiental que rige sus actividades"27.

\footnotetext{
${ }^{25}$ TRIBUnAl CONSTitucional. Sentencia recaída en el Expediente $\mathrm{N}^{\circ}$ 4223-2006-PA/TC, FJ 34.

${ }^{26}$ Tribunal ConstituCIONAL. Sentencia recaída en el Expediente N ${ }^{\circ}$ 4223-2006-PA/TC, FJ.35.

${ }^{27}$ Tribunal ConstituCiONAL. Sentencia recaída en expediente $\mathrm{N}^{\circ}$ 3196-2004-AC/TC, FJ. 6.
} 
En efecto, el principio precautorio debe fundamentarse no sólo en una duda razonable sobre la supuesta violación de derechos constitucionalmente protegidos; sino que requiere de un test mínimo de razonabilidad o proporcionalidad consagrado en la jurisprudencia constitucional en el cual se realizan los tres sub juicios: adecuación, necesidad; proporcionalidad en sentido estricto ${ }^{28}$.

En líneas generales, consideramos prima facie que la protección del medio ambiente como parámetro de validez de las actividades económicas debe tener al principio precautorio desde una perspectiva minimalista, y por ende, opuesta a la maximalista. Lo que supone que el Estado lleve a cabo tareas de supervisión, fiscalización y sanción; pero, siempre de manera razonable y proporcional. Así, por ejemplo, muchos países han incorporado los llamados "tributos ambientales", siguiendo el principio contaminador-pagador, en virtud el cual "permiten internalizar las externalidades negativas con el fin de equilibrar los costes sociales que genera la contaminación y degradación ambiental"29.

Sobre el particular, en el Perú se dictó la Ley № 28694 (2006) que estableció medidas tributarias para el uso de combustibles limpios, estableciendo el criterio de proporcionalidad al grado de nocividad por los contaminantes que éstos contengan para la salud de la población. Para tal efecto el Estado debe aprobar anualmente los índices de nocividad relativa a ser utilizados; no obstante, los gobiernos desde hace una década han ralentizado su aplicación. Asimismo, en un concepto amplio de "tributo ambiental" se podría mencionar el establecimiento de la tasa por el uso del agua subterránea de los acuíferos de Lima y Callao ${ }^{30}$.

Ahora bien, un escrutinio administrativo y/o judicial sobre la aplicación del principio precautorio debería incorporar distintos grados de intensidad de control estatal sobre la actividad bajo observación y control, en función de ciertas presunciones del riesgo y del manejo de la inversión de la carga de la prueba. Así, se podría postular una aplicación del principio de precaución: débil, moderada y fuerte; en función del tipo de riesgos ciertos o inciertos, que abren las opción a evitar el mismo, o, en última instancia gestionar el riesgo. Pero, el Tribunal de Fiscalización Ambiental del Organismo de Evaluación y Fiscalización Ambiental (OEFA) no ha desarrollado en la motivación de sus resoluciones elementos argumentativos del principio de precaución, como para construir y aplicar un test de la precaución ${ }^{31}$.

De acuerdo con el modelo constitucional peruano opera la presunción fuerte; donde el causante del riesgo debe demostrar la inocuidad de su actividad. En concreto, se puede decir que existe una tendencia a establecer que en primera instancia se ha abandonado la regla "es seguro mientras no se demuestre que es perjudicial". Pero, la presunción genérica de inocuidad no es posible de mantener en un contexto como en el siglo XX y XXI en el que se ha descubierto la toxicidad de numerosos productos parecían inocuos ${ }^{32}$. Por eso, es necesario analizar el principio de prevención.

\subsection{Principio de Prevención}

El principio de prevención se aplica en la gestión ambiental, tiene como objetivos prioritarios prevenir, vigilar y evitar la degradación ambiental; pero, cuando no sea posible

\footnotetext{
${ }^{28}$ Tribunal Constitucional. Sentencia recaída en el expediente $N^{\circ}$ 02005-2009-AA. Voto de los magistrados Landa Arroyo y Calle Hayen, FJ. 49.

${ }^{29}$ FigueroA Neri, Aimée. Fiscalidad y Medio Ambiente en México. México: Porrúa, 2000, p. 45.

30 PATRÓN GARCÍA, Gemma. Fiscalidad ambiental, responsabilidad social y desarrollo sostenible en América Latina. Propuesta para Perú. Lima: Thomson Reuters, 2016, pp. 97-103.

${ }^{31}$ Tribunal DE FisCALIZACión AmBIEnTAL. Jurisprudencia Ambiental. OEFA. En http://www.oefa.gob.pe/?wpfb dl=19031. Visitado el 18 de octubre de 2016.

${ }^{32}$ TICKNER, Joel. "Un mapa hacia la toma de decisiones precautoria". En RIECHMANN, Jorge y Tickner, Joel (coordinadores). El principio de precaución en medio ambiente y salud pública: de las definiciones a la práctica. Barcelona: Icaria, 2002, p. 51.
} 
eliminar las causas que la generan, se adoptan las medidas de mitigación, recuperación, restauración o eventual compensación que correspondan, señala la Ley General de Ambiente (artículo VI).

La regla riesgo-beneficio también se encuentra estrechamente ligada al principio de prevención. De hecho, en la teoría tradicional, el principio de prevención es el que se aplica para evitar que un riesgo comprobado genere un daño o una afectación al medio ambiente o a la salud de las persona.

En efecto, cabe reiterar que el principio de prevención se aplica en el supuesto en que el riesgo se encuentra acreditado, pero se acuerda un nivel aceptable respecto del mismo. La diferencia con el principio precautorio es que este último se aplica en los casos en los que existe una incertidumbre sobre el riesgo, es decir, cuando hay una probabilidad de un riesgo con consecuencias poco conocidas.

Ahora bien, la obligación de señalar un riesgo determinado o, lo que es lo mismo, la imposibilidad de legitimar medidas precautorias ante peligros abstractos, obliga desde esta perspectiva a una distinción entre precaución y prevención no basada en la distinción entre riesgo hipotético y riesgo potencial, o entre posibilidad y probabilidad, sino entre riesgos potenciales afirmados más o menos unánimemente por la comunidad científica y riesgos potenciales sobre los que existe disenso científico o incertidumbre ${ }^{33}$.

Asimismo, en el caso del principio de prevención se podría decir que tiene una relación directa con el principio de "diligencia debida", a partir del cual se busca adoptar previsiones para asegurarse que, en condiciones normales, los objetos, elementos o actividades riesgosas no causen perjuicios a terceros ${ }^{34}$.

En el caso de las licencias y permisos para la explotación de actividades mineras y petroleras, el principio de prevención cobra especial relevancia, ya que este es plenamente aplicable a la relación riesgo-beneficio; dada lo regular de la contaminación ambiental ${ }^{35}$. Pero, en la práctica en la explotación de los recursos naturales se requiere que el beneficio de su empleo sea superior a los riesgos que pueda generar; en la práctica, los permisos de la mayor parte de las empresas mineras y petroleras implican un cierto riesgo que debe tratar de reducirse al máximo.

Así el Tribunal de Fiscalización Ambiental ha aprobado una resolución como precedente administrativo de observancia obligatoria, mediante la cual se establece que el artículo 5 del Reglamento para la Protección Ambiental en la Actividad Minero-Metalúrgica, aprobado por el Decreto Supremo $\mathrm{N}^{\circ}$ 016-93-EM, impone al titular minero dos obligaciones: 1) adoptar con carácter preventivo las medidas necesarias para evitar e impedir que las emisiones, vertimientos, desechos, residuos u otros que se produzcan como resultado de las actividades realizadas o situaciones generadas en sus instalaciones puedan tener efectos adversos en el ambiente, sin que sea necesaria la verificación ambienta, y; 2) no exceder los límites máximos permisibles ${ }^{36}$.

Asimismo, ante la falta de adopción de medidas de prevención y control debido al incremento de las lluvias que ocasionó que las aguas de una laguna se desbordara llegando a un centro minero y entrara en contacto con los minerales oxidados expuestos y los afloramiento de sulfuros, arrastrando metales pesados en su curso de retorno hasta la laguna, el Tribunal de

\footnotetext{
${ }^{33}$ Montoro Chiner, María Jesús. "Perspectivas de contraste ante el riego ecológico. Agentes que interviene en la evaluación del riesgo. En especial, los comités científicos". En Riesgo y Precaución. Hacia una bioética ambiental. Residència d'Investigadors CSIC-Generalitat de Catalunya Barcelona, 2005, pp. 125-136.

${ }^{34}$ ORGANIZACIÓN DE ESTADOS AMERICANOS. "Los principios de prevención y precaución en materia ambiental en el sistema internacional y en el interamericano”. En: Jornadas de Derecho Internacional, Secretaría General de la OEA, Washington, 2001, pp. 81-92.

${ }^{35}$ CLÉMENT, Zlata Drnas de. El "Principio de Precaución en Materia Ambiental. En: Academia Nacional de Derecho y Nuevas Tendencias. Disponible en: http://www.acader.unc.edu.ar/artpricipio deprecaucion.rtf. Revisado el 20 de octubre de 2016.

${ }^{36}$ TRIBUNAL DE FiSCALIZACiÓn AMBIENTAL. Resolución No 021-2014-OEFA/TFA-SEPI del 30 de octubre de 2014. Revista de Direito Brasileira | São Paulo, SP | v. 16 | n. 7 | p. 412 - 427 | Jan./Abr. 2017
} 
Fiscalización Ambiental multó a una empresa por incumplir el Reglamento Ambiental para las Actividades de Exploración Minera (Decreto Supremo N 020-2008-EM) ${ }^{37}$.

En efecto, frente a los riesgos comprobados le sigue el principio de gestión del riesgo que se deriva del propio principio de prevención y que consiste en sopesar los riesgos y los beneficios derivados de una actividad extractiva potencialmente contaminante, y seleccionar estrategias que modifiquen dichos niveles. En ese sentido, se debería tener en cuenta una serie de pasos o principios sobre la materia, que siguiendo a Sánchez se resume de la siguiente manera:

a) Proporcionalidad de las medidas que se adopten al nivel de protección deseado, teniendo en cuenta que jamás se puede alcanzar un riesgo cero;

b) No discriminación, en virtud de la cual situaciones comparables no han de tratarse de manera diferente y situaciones diferentes no deben tratarse de la misma forma;

c) Consistencia de las medidas, por cuanto tienen que ser comparables a las que se han adoptado en circunstancias similares o utilizando enfoques parecidos;

d) Estudio de los beneficios y los costes de las acciones y de la falta de acción; esto es realizar un análisis del coste-beneficio, de la eficacia, del impacto económico y social y, en determinadas circunstancias, consideraciones no economicistas $^{38}$.

En el marco del proceso de gestión de riesgos, también debe regir el principio de transparencia que permita tanto al Estado como a la sociedad encontrarse informados, pero también que las empresas extractivas gocen de mayor credibilidad y legitimidad en tanto cogarantes del derecho al medio ambiente; en la medida que la sociedad acepta muchas situaciones que suponen, a la vez, riesgos y beneficios ${ }^{39}$.

En concreto, en materia ambiental, el principio de prevención involucra que en los planes de gestión de riesgos incorporen en su contenido el modo en que el riesgo de contaminación debe ser prevenido o minimizado; realice estudios o actividades para profundizar el conocimiento sobre la seguridad ambiental de la actividad extractiva y/o transformadora; también si existen factores de riesgos para desarrollar los efectos adversos; así como la evaluación de las medidas de las actividades de minimización de los riesgos.

Asimismo, conviene señalar que el plan de gestión de riesgos es una obligación que deben cumplir los titulares que cuentan con una licencia o autorización para explorar y explotar recursos naturales, sobretodo minero y energético; los cuales deben presentar a la agencia regulatoria del Estado, competente en la materia, información pertinente respecto de la gestión de riesgos. En esa lógica, el principio de prevención también implica que la autoridad administrativa tenga el deber de exigir la actualización del plan de gestión de riesgos de acuerdo a la nueva información de las actividades extractivas, de transformación o manufacturación.

En efecto, el Tribunal de Fiscalización Ambiental en el caso de una empresa minera estableció que la obligación de prevención se encuentra directamente dirigida a que el titular minero adopte no solo un plan de manejo ambiental, sino también otras medidas necesarias con la finalidad de evitar que se afecte negativamente al ambiente con todas aquellas actividad que se generan al interior de la concesión minera, más allá de que se superen o no los límites máximos permisibles $^{40}$.

De otro lado, el principio de prevención implica que en base a la identificación de los riesgos comprobados se implementen acciones o medidas regulatorias de acuerdo a su

\footnotetext{
${ }^{37}$ TRIBUNAL DE FisCALIZACIÓN AMBIENTAL. Resolución Nº 044-2015-OEFA/TFA-SEPI del 1 de julio de 2015.

38 SÁNCHEZ, Emilia. "El principio de precaución: implicaciones para la salud pública". En Gaceta Sanitaria, volumen 16, $\mathrm{N}^{\circ} 5,2002$, pp. 371-373.

${ }^{39}$ Ídem.

${ }^{40}$ TRIBUNAL DE FISCALIZACión AMBIENTAL. Resolución N 005-2015-OEFA/TFA-SEPI del 13 de enero de 2015. Revista de Direito Brasileira | São Paulo, SP | v. 16 | n. 7 | p. 412 - 427 | Jan./Abr. 2017
} 
aceptabilidad. Así, las medidas administrativas de riesgo involucran una gradación en la gestión de los mismos. En concreto, siguiendo subsidiariamente los criterios panamericanos de protección de la salud pública, se puede hacer referencia a tres tipos de riesgo: el riesgo aceptable en las condiciones de uso; el riesgo aceptable en ciertas condiciones; el riesgo inaceptable en cualquier situación y en función de ello, las medidas administrativas serían medidas de prevención, como la necesidad de informar, restringir ciertas actividades, y, el retiro del permiso, respectivamente ${ }^{41}$.

A partir de lo mencionado, se puede señalar que el principio de prevención también permite gradaciones en cuanto a la aprobación u otorgamiento de una licencia o registro de actividades extractivas sobre recursos naturales. Así, dependiendo del grado de peligro o riesgo comprobado se podrá admitir o no el permiso solicitado. Ahora bien, en el caso de los riesgos y/o peligros comprobados estos deben ser menores en comparación con los beneficios de uso del mismo.

Ahora, como se trata de limitar derechos que están inmersos en una pluralidad de normas e interpretaciones, el Tribunal Constitucional ha establecido que debe optarse siempre "por aquella norma ius fundamental que garantice de la manera más efectiva y extensa posible los derechos fundamentales reconocidos; es decir aquella que despliegue una mayor eficacia de la norma" 42 .

Para lo cual el test de proporcionalidad debe ser el instrumento aplicable en situaciones de incertidumbre y de riesgo comprobado. En efecto, el examen de proporcionalidad es una técnica que permite resolver conflictos entre principios de orden constitucional. Pero, trasladado al ámbito del medio ambiente, el examen en mención permitirá determinar si prevalece el riesgo o el beneficio de una actividad que pueda ser contaminante. De hecho, en las medidas de protección ambiental en base al principio precautorio, la relación riesgo-beneficio debe determinarse en función de la razonabilidad o proporcionalidad.

El principio de proporcionalidad se usa para valorar la legitimidad de una medida restrictiva de la Autoridad Ambiental respecto a la limitación en el ejercicio de la libertad de empresa en la actividad extractiva, transformadora o comercializadora de recursos naturales ${ }^{43}$. Ahora bien, el principio de proporcionalidad permite determinar, a partir de tomar medidas menos lesivas, el monto de una multa u otra sanción, la denegatoria de un registro o al establecer límites regulatorios a la explotación de un recurso natural, como los mineros - energéticos; como podría ser, por ejemplo, prohibir el uso de mercurio en la explotación del oro, dado el altísimo grado de contaminación ambiental que produce en caso de accidentes.

En concreto, cuando se realiza un examen de proporcionalidad, la medida será proporcional si supera los tres pasos del mismo: Test de idoneidad; test de necesidad, y; test de proporcionalidad en sentido estricto.

$>\quad$ El test de idoneidad: La idoneidad consiste en la relación de causalidad, de medio a fin, entre el medio adoptado, a través de la intervención legislativa, y el fin propuesto por el legislador. Se trata del análisis de una relación medio-fin $(\ldots)^{44}$.

$>\quad$ El test de necesidad: Bajo este test ha de analizarse si existen medios alternativos al optado por el legislador que no sean gravosos o, al menos, que lo

\footnotetext{
${ }^{41}$ Red PANAMERICANA PARA LA ARMONIZACIÓN DE LA REGLAMENTACIÓN FARMACÉUTICA. Planes de gestión de riesgo para las Américas, Washington, diciembre de 2013. En file:///D:/Users/clanda/Downloads/ Consulta_planes_de_gestion_de_riesgo\%20(1).pdf. Visitado el 18 de octubre de 2016.

${ }_{42}^{42}$ TRIBUNAL CONSTITUCIONAL. Sentencia recaída en expediente $\mathrm{N}^{\circ}$ 02005-2009-AA/TC. FJ 33.

43 MARTíNEZ PÉREZ, Enrique. "La delimitación jurisprudencial comunitaria de los requisitos para la aplicación del principio de precaución". Disponible en: http://consumo-inc.gob.es/publicac/EC/ 2003/EC67/EC67_1.pdf, revisado el 20 de octubre de 2016.

${ }^{44}$ Tribunal Constitucional. Sentencia recaída en expediente $\mathrm{N}^{\circ}$ 0045-2005-AI/TC, FJ.38
}

Revista de Direito Brasileira | São Paulo, SP | v. 16 | n. 7 | p. 412 - 427 | Jan./Abr. 2017 
sean en menor intensidad. Se trata del análisis de una relación medio-medio, esto es, de una comparación entre medios; el optado por el legislador y el o los hipotéticos que hubiera podido adoptar para alcanzar el mismo fin $(\ldots)^{45}$.

$>\quad$ El test de proporcionalidad en sentido estricto: (...) consistirá en una comparación entre el grado de realización u optimización del fin constitucional y la intensidad de la intervención en la igualdad. La comparación de estas dos variables ha de efectuarse según la denominada ley de ponderación. Conforme a ésta: Cuanto mayor es el grado de la no satisfacción o de la afectación de un principio, tanto mayor tiene que ser la importancia de la satisfacción del otro $(\ldots)^{46}$.

En líneas generales, en el examen que se realice respecto de si se otorga o no un permiso o licencia minero o hidrocarburífero, ya sea que se trate de un examen basado en el principio de prevención o incluso precautorio, en todo caso, deben plantearse medidas o soluciones que admitan una graduación dependiendo del tipo de riesgo frente al cual se debe tomar una decisión.

En esa perspectiva, el examen de proporcionalidad en el que se analizan los beneficios y los riesgos de una actividad minera-energética, debiera realizarse sobre la base de criterios cuantitativos y no solo cualitativos, a fin de que en aplicación de los principios de precaución o prevención prime la racionalidad o la objetividad antes que la subjetividad de la autoridad decisora $^{47}$.

\section{CONCLUSIÓN}

El Perú es un país mega-diverso y pluricultural con una riqueza de recursos naturales renovables y no renovables, con bosques poblados de especies animales y vegetales únicos, pero inmerso frágilmente en la cresta del cambio climático global; donde el Estado como garante del interés general tiene la obligación de construir un sistema de justicia ambiental.

En la búsqueda de la justicia ambiental la jurisprudencia constitucional y administrativa encierra un potencial de desarrollo dentro de la Constitución Ambiental, dado el imperativo de la argumentación a partir de pensamiento constitucional verde que guarde correspondencia con su entorno ambiental ${ }^{48}$. En tal sentido, se puede decir que la ética ambiental sobre la cual se viene construyendo la Constitución Ecológica es de naturaleza antropocéntrica, en la medida que la defensa de la persona humana y el respeto a su dignidad es el fin supremo de la sociedad, del Estado y del mercado.

En ese sentido, no solo la Constitución y la legislación ambiental, sino también la jurisprudencia nacional e internacional se orienta hacia el bienestar del ser humano. Así, en sociedades opulentas en riquezas y bio-diversidad corresponde al Estado distribuir adecuadamente los derechos, deberes y obligaciones para el logro de un ambiente equilibrado; pero, este proceso de desarrollo sostenible no está exento de promover la protección de bienes jurídicos especiales que nos brinda la naturaleza, como a través de los derechos de los animales ${ }^{49}$.

Esta perspectiva ecológica de no instrumentalizar la naturaleza al servicio del hombre, sino respetarla por derecho propio; se pone en evidencia cuando las poblaciones indígenas asentadas en territorios andinos o amazónicos ricos en recursos naturales, se sienten afectadas por las empresas mineras y petroleras en su derecho consuetudinario ancestral, debido a que conciben

\footnotetext{
${ }^{45}$ Ibídem, FJ. 39.

${ }^{46}$ Ibídem, FJ. 40.

${ }^{47}$ EMBID Tello, Antonio...., op. cit.

${ }^{48}$ Sarlet, Ingo; FenSTERSEIFER, Tiago. Direito Constitucional Ambiental. Constituição, Direitos Fundamentais e Proteção do Ambiente. Sao Paulo: Revista dos Tribunais, 2014, pp. 41-45.

${ }^{49}$ Tribunal Constitucional. Sentencia recaída en expediente No 00042-2004-AI/TC, FJ 27.
}

Revista de Direito Brasileira | São Paulo, SP | v. 16 | n. 7 | p. 412 - 427 | Jan./Abr. 2017 
su comunidad como parte integrante de la naturaleza física y espiritual (ríos, montañas, etc.) formando así su identidad cultural ${ }^{50}$.

En ese escenario los postulados constitucionales clásicos requieren revisarse bajo un horizonte temporal de largo plazo mediante un nuevo pacto constitucional ambiental conforme a los intereses del presente que integre a las poblaciones indígena, sino también de las futuras generaciones, y, con respeto a la naturaleza ${ }^{51}$. Por ello, esta es una responsabilidad de la sociedad y no solo del Estado, porque, cuando éste ha asumido la forma del Estado de Derecho ha servido más a intereses privados que al interés general. Por eso, solo bajo la forma del nuevo Estado Constitucional participativo es que existe la posibilidad de reintegrar éticamente a toda la comunidad, bajo el ethos de la Constitución Ambiental y/o Ecológica ${ }^{52}$.

\section{REFERENCIAS}

Acuerdo de Paris sobre Cambio Climático de la Convención Marco de las Naciones Unidas sobre el Cambio Climático. En: 〈http://unfccc.int/resource/docs/2015/cop21/spa/109s.pdf〉. Visitado el 1 de octubre de 2016.

AL GoRE. Una verdad incómoda. En: <http://es.slideshare.net/edau1/una-verdad-incmoda-algore>. Visitado el 3 de octubre de 2016.

BECK, Ulrich. La sociedad del riesgo: hacia una nueva modernidad. Bracelona: Paidós, 2001.

Ben LAZAAR, Soufiane. El principio de precaución en el derecho internacional del medio ambiente. Trabajo de Fin de Máster, Universidad Internacional de Andalucía, 2012. En: <http://dspace.unia.es/bitstream/handle/10334/2117/0256_BenLazaar.pdf?sequence=1>.

Revisado el 01 de octubre de 2016.

Clément, Zlata Drnas de. El Principio de Precaución en Materia Ambiental. En: Academia Nacional de Derecho y Nuevas Tendencias. En: <http://www.acader.unc.edu.ar/artpricipio deprecaucion.rtf $>$. Revisado el 20 de octubre de 2016.

COMisión Mundial de Ética del CONOCIMIENTO Científico Y la TeCNOlogía (COMEST). INFORME DEL GRUPO DE EXPERTOS SOBRE EL PRINCIPIO PRECAUTORIO. PARIS, UNESCO, 2005. En: <http://unesdoc.unesco.org/ images/0013/001395/139578s.pdf $>$. Visitado el 15 de octubre de 2016.

Conferencia de las Naciones Unidas sobre el Medio Ambiente y el Desarrollo. Declaración de Rio sobre el Medio Ambiente y el Desarrollo. En: <http://www.pnuma.org/sociedad_civil/reunion2013/docu mentos/STAKEHOLDER\%20PARTICIPATION/1992\%20Declaraci\%C3\%B3n\%20de\%20R\%C 3\%ADo\%20Espa\%C3\%B1ol.pdf>. Visitado el 10 de octubre de 2016.

Corte Interamericana de Derechos Humanos. Caso Pueblo Indígena Kiwcha de Sarayaku vs. Ecuador. Sentencia de 27 de junio de 2012.

. Caso del Pueblo Saramaka vs. Surinam. Sentencia del 28 de noviembre de 2007.

\footnotetext{
${ }^{50}$ LANDA, César. Derechos colectivos de los pueblos indígenas u originarios. Lima: Ministerio de Cultura, 2016, pp. 61.

${ }^{51}$ HÄBERLE, Peter. Das ewige Ringen um die Gerechtigkeit. En Forschung \& Lehre, 2 / 98, pp. 86-88.

${ }^{52} \mathrm{DE}$ CABO, Carlos. Contra el consenso, estudios sobre el Estado constitucional y el constitucionalismo del Estado social. México: Universidad Autónoma de México, 1997, pp. 317 ss.
}

Revista de Direito Brasileira | São Paulo, SP | v. 16 | n. 7 | p. 412 - 427 | Jan./Abr. 2017 
DE CABo, Carlos. Contra el consenso, estudios sobre el Estado constitucional y el constitucionalismo del Estado social. México: Universidad Autónoma de México, 1997.

EMBID Tello, Antonio. Retos jurídicos de la radiación no ionizante, Tesis doctoral, Madrid, Universidad Carlos III. En: < $\underline{\text { http://e-archivo.uc3m.es/bitstream/handle/10016/7622 }}$ lantonio_embid

tesis.pdf;jsessionid=D4B659855A6F6E75F9B68B6ECEEB7FEC?sequence=1>. Visitado el 10 de octubre de 2016.

En la actualidad también se aplica al ámbito del derecho a la salud y a la salud pública. En: <http://www.bioscience-bioethics.org/pdfs/NW.Public.Health.PP.2005.pdf $>$. Visitado el 11 de octubre de 2016.

Foy Valencia, Pierre. Constitucionalidad del Desarrollo Sostenible. Lima, marzo 2014, inédito.

INFORME BRUNTLAND. En: <https://desarrollosostenible.wordpress.com/2006/09/27/informebrundtl and/>. Visitado el 10 de octubre de 2016.

Figueroa Neri, Aimée. Fiscalidad y Medio Ambiente en México. México: Porrúa, 2000.

HäBERLE, Peter. Ein Verfassungsrecht für künftige Generationen. Die “andere” Form des Gesellschaftsvertrages: der Generationenvertrag. . Verfassung, Theorie und Praxis des Sozialstaats. Festschrift für Hans F. Zacher zum 70. Geburtstag, 1998. . Retos actuales del Estado Constitucional. Oñati: IVAP, 1996. . Das ewige Ringen um die Gerechtigkeit. En Forschung \& Lehre, 2/98.

Huerta Guerrero, Luis. Constitucionalización del derecho ambiental. En Derecho PUCP 71. Lima: Fondo Editorial PUCP, 2013.

LANDA, César. Derechos colectivos de los pueblos indígenas u originarios. Lima: Ministerio de Cultura, 2016.

Ley $\mathrm{N}^{\circ}$ 28611, consagra en su Título Preliminar, los principios de sostenibilidad, prevención, precautorio, de internacionalización de costos, de responsabilidad ambiental, de equidad y de gobernanza ambiental; además del derecho a la justicia ambiental, participación y acceso a la información.

MARTÍNEZ PÉREZ, Enrique. La delimitación jurisprudencial comunitaria de los requisitos para la aplicación del principio de precaución. En: <http://consumo-inc.gob.es/publicac/EC/ 2003/EC67/EC67_1.pdf>. Revisado el 20 de octubre de 2016.

Montoro Chiner, María Jesús. Perspectivas de contraste ante el riego ecológico. Agentes que interviene en la evaluación del riesgo. En especial, los comités científicos. En Riesgo y Precaución. Hacia una bioética ambiental. Residència d'Investigadors CSIC-Generalitat de Catalunya Barcelona, 2005.

ORGANIZACIÓN DE ESTADOS AMERICANOS. Los principios de prevención y precaución en materia ambiental en el sistema internacional y en el interamericano. En: Jornadas de Derecho Internacional. Secretaría General de la OEA. Washington, 2001. 
PATRÓN GARCÍA, Gemma. Fiscalidad ambiental, responsabilidad social y desarrollo sostenible en América Latina. Propuesta para Perú. Lima: Thomson Reuters, 2016.

Programa de Naciones Unidas Para el Medio AmbIEnTE. Medio ambiente para el futuro que queremos. En: <http://www.pnuma.org/geo/geo5/GEO\%205\%20ESPANOL \%202013\%20WEB.pdf >. Visitado el 3 de octubre de 2016.

RED PANAMERICANA PARA LA ARMONIZACIÓN DE LA REGLAMENTACión FARMACÉUTiCA. Planes de gestión de riesgo para las Américas. Washington, diciembre de 2013. En <file:///D:/Users/clanda/Downloads/ Consulta_planes_de_gestion_de_riesgo\%20(1).pdf>. Visitado el 18 de octubre de 2016.

Saladin, Peter. Wozu noch Saaten? Berna: Verlag Stämpfli+Cie AG, 1995.

SÁNCHEZ, Emilia. El principio de precaución: implicaciones para la salud pública. En: Gaceta Sanitaria, volumen 16, № 5, 2002.

SARlet, Ingo; Fensterseifer, Tiago. Direito Constitucional Ambiental. Constituição, Direitos Fundamentais e Proteção do Ambiente. Sao Paulo: Revista dos Tribunais, 2014.

TICKNER, Joel. Un mapa hacia la toma de decisiones precautoria. En RIECHMANN, Jorge y Tickner, Joel (coordinadores). El principio de precaución en medio ambiente y salud pública: de las definiciones a la práctica. Barcelona: Icaria, 2002.

TRIBUnAl Constitucional. Sentencia recaída en el Expediente $N^{\circ}$ 3610-2008-AA. . Sentencia recaída en el Expediente 03343-2007-PA. . Sentencia recaída en el Expediente $N^{\circ} 0048-2004-P I / T C$. . Sentencia recaída en el Expediente $\mathrm{N}^{\circ}$ 06316-2008-PA/TC. Voto singular del Magistrado Landa Arroyo.

. Sentencia recaída en el Expediente $\mathrm{N}^{\circ}$. 03343-2007-PA/TC. Sentencia recaída en el Expediente $\mathrm{N}^{\circ}$ 0964-2002-AA/TC. . Sentencia recaída en el Expediente $\mathrm{N}^{\circ}$ 4223-2006-PA/TC. Sentencia recaída en expediente $\mathrm{N}^{\circ}$ 3196-2004-AC/TC. Sentencia recaída en el expediente $\mathrm{N}^{\circ}$ 02005-2009-AA. Voto de los magistrados Landa Arroyo y Calle Hayen.

. Sentencia recaída en expediente $\mathrm{N}^{\circ}$ 00042-2004-AI/TC. . Sentencia recaída en expediente $\mathrm{N}^{\circ}$ 0045-2005-AI/TC.

TRIBUNAL DE FISCALIZACIÓN AMBIENTAL. Jurisprudencia Ambiental. OEFA. En: <http://www.oefa.gob.pe/?wpfb_dl=19031>. Visitado el 18 de octubre de 2016.

. Resolución N 021-2014-OEFA/TFA-SEPI del 30 de octubre de 2014.

Resolución N 044-2015-OEFA/TFA-SEPI del 1 de julio de 2015.

. Resolución $N^{\circ}$ 005-2015-OEFA/TFA-SEPI del 13 de enero de 2015.

Tribunal de Justicia de la Comunidad Europea. Caso Pfizer Animal Health, del 11 de septiembre de 2002, asunto T-13/99, o Caso Monsanto Agricultura Italia y otros, de 9 de septiembre de 2003, asunto C-236/00. En: 〈http://curia.europa.eu.> Visitado 10 de octubre de 2016. 